\title{
Avaliação agronômica de genótipos de amendoim no Médio Norte do estado do Mato Grosso
}

\author{
Submetido - 02 Jul. 2021 \\ Aprovado - 31 jul. 2021 \\ Publicado - 30 set. 2021
}

http://dx.doi.org/10.52755/sas.v.2i(edesp1)123

\begin{abstract}
Dácio Olibone (iD)
Professor do Instituto Federal de Educação, Ciência e Tecnologia de Mato Grosso - IFMT, Sorriso, MT.E-mail: dacio.olibone@srs.ifmt.edu.br.
\end{abstract}

Programa de Melhoramento do Amendoim - Embrapa, Santo Antônio de Goiás, GO. E-mail: jair.heuert@embrapa.br.

Ana Paula Encide Olibone (D) Professora do Instituto Federal de Educação, Ciência e Tecnologia de Mato Grosso - IFMT, Sorriso, MT. E-mail: ana.olibone@srs.ifmt.edu.br.

Discente de Engenharia Agronômica, Instituto Federal de Educação, Ciência e Tecnologia de Mato Grosso - IFMT, Sorriso, MT. E-mail: marianithamariano@gmail.com.

Discente de Engenharia Agronômica, Instituto Federal de Educação, Ciência e Tecnologia de Mato Grosso - IFMT, Sorriso,MT. E-mail: sergios7soares@gmail.com.

Taís de Moraes Falleiro Suassuna $\mathbb{D}$ Programa de Melhoramento do Amendoim - Embrapa, Santo Antônio de Goiás, GO. E-mail: tais.suassuna@embrapa.br.

\section{RESUMO}

Este trabalho foi desenvolvido com o objetivo de realizar uma avaliação agronômica de genótipos de amendoim no Médio Norte do estado do Mato Grosso. 0 experimento foi desenvolvido no ano agrícola 2020/21 com semeadura manual no dia 06 de janeiro de 2021, na área experimental do Instituto Federal de Educação, Ciência e Tecnologia de Mato Grosso Campus Sorriso, localizado no município de Sorriso-MT. O delineamento experimental foi em blocos casualizados, com quatro repetições. Os tratamentos foram dez genótipos de amendoim rasteiro, duas cultivares: BRS 421 OL, BRS 423 OL e oito linhagens: 1253 OL, 2055 OL, 2091 OL, 2101 OL, 2110 OL, 2133 OL, 2136 OL e 2259 OL, desenvolvidas pelo PMA da EMBRAPA. As parcelas foram compostas por duas linhas de três metros de comprimento, espaçamento entre linhas de 0,90 m, intervalo entre parcelas de dois metros e parcela útil 5,4 $\mathrm{m}^{2}$. Foram avaliados severidade de mancha preta (Cercosporidium personatum), massa de 100 grãos (g), rendimento (\%) e produtividade de vagens ( $\mathrm{kg} \mathrm{ha}^{-1}$ e sacas alqueire ${ }^{-1}$ ). Houve diferença significativa entre os genótipos avaliados para severidade de mancha preta, massa de 100 grãos, rendimento e produtividade. Os menores valores de severidades foram observados nas cultivares BRS 421 OL e BRS 423 OL, sendo que as sementes de maior tamanho também foram observadas na BRS 421 OL. As maiores produtividades foram obtidas no BRS 421 OL, 2101 OL, 2259 OL, 2055 OL e 1253 OL, com variável tamanho de sementes entre estes genótipos.

Palavras-chave: Arachis hypogaea L.; Amendoim em Mato Grosso; Programa de Melhoramento; Produtividade.

Este é um trabalho de acesso aberto e distribuído sob os Termos da Creative Commons Attribution Attribution-NonCommercial-ShareAlike 4.0 International. 


\title{
Agronomic evaluation of peanut genotypes in northern Mato Grosso state
}

\begin{abstract}
This work was developed with the objective of carrying out an agronomic evaluation of peanut genotypes in the Middle North of the state of Mato Grosso. The experiment was carried out in the agricultural year 2020/21 with manual sowing on January 6, 2021, in the experimental area of the Federal Institute of Education, Science and Technology of Mato Grosso - Campus Sorriso, located in the municipality of Sorriso-MT. The experimental design was in randomized blocks, with four replications. The treatments were ten groundnut genotypes, two cultivars: BRS 421 OL, BRS 423 OL and eight lines: 1253 OL, 2055 OL, 2091 OL, 2101 OL, 2110 OL, 2133 OL, 2136 OL and 2259 OL, developed by PMA of EMBRAPA. The plots were composed of two lines of three meters in length, spacing between lines of $0.90 \mathrm{~m}$, interval between plots of two meters and useful plot of $5.4 \mathrm{~m}^{2}$. Black spot severity (Cercosporidium personatum), mass of 100 grains (g), yield (\%) and pod yield (kg ha-1 and bushel sacks $\left.{ }^{-1}\right)$ were evaluated. There was a significant difference between the genotypes evaluated for black spot severity, 100 grain mass, yield and yield. The lowest values of severities were observed in cultivars BRS 421 OL and BRS $423 \mathrm{OL}$, and the seeds of larger size were also observed in BRS $421 \mathrm{OL}$. The highest yields were obtained in BRS 421 OL, 2101 OL, 2259 OL, 2055 OL and 1253 OL, with variable seed size among these genotypes.
\end{abstract}

Keywords: Arachis hypogaea L.; Peanut in Mato Grosso; Improvement Program; Productivity.

Evaluación agronómica de genotipos de maní en el norte del estado de Mato Grosso

\section{RESUMEN}

Este trabajo se desarrolló con el objetivo de realizar una evaluación agronómica de genotipos de maní en el Medio Norte del estado de Mato Grosso. El experimento se realizó en el año agrícola 2020/21 con siembra manual el 6 de enero de 2021, en el área experimental del Instituto Federal de Educación, Ciencia y Tecnología de Mato Grosso - Campus Sorriso, ubicado en el municipio de Sorriso-MT. El diseño experimental fue en bloques al azar, con cuatro repeticiones. Los tratamientos fueron diez genotipos de maní, dos cultivares: BRS 421 OL, BRS 423 OL y ocho líneas: 1253 OL, 2055 OL, 2091 OL, 2101 OL, 2110 OL, 2133 OL, 2136 OL y 2259 OL, desarrollados por PMA de EMBRAPA. Las parcelas estaban compuestas por dos líneas de tres metros de longitud, espaciamiento entre líneas de $0,90 \mathrm{~m}$, intervalo entre parcelas de dos metros y parcela útil de $5,4 \mathrm{~m}^{2}$. Se evaluó la severidad de la mancha negra (Cercosporidium personatum), masa de 100 granos (g), rendimiento (\%) y rendimiento de vaina (kg ha-1 y bushel sacks ${ }^{-1}$ ). Hubo una diferencia significativa entre los genotipos evaluados para la severidad de la mancha negra, masa de 100 granos, rendimiento y rendimiento. Los valores más bajos de severidad se observaron en los cultivares BRS 421 OL y BRS 423 OL, y las semillas de mayor tamaño también se observaron en BRS 421 OL. Los mayores rendimientos se obtuvieron en BRS 421 OL, 2101 OL, 2259 OL, 2055 OL y 1253 OL, con tamaño de semilla variable entre estos genotipos.

Palabras clave: Arachis hypogaea L.; Maní en Mato Grosso; Programa de Mejoramiento; Productividad.

\section{Introdução}

A cultura do amendoim (Arachis hypogaea L.) vem ganhando espaço no Estado de Mato Grosso e atraindo novos produtores. Nas últimas safras, o estado, principalmente na região Médio Norte, apresentou um 
grande crescimento na área cultivada com a leguminosa, que é uma ótima alternativa de cultivo. Dados experimentais no município de Sorriso-MT, desde a safra 2018/19 têm demostrado que a cultura tem boa adaptação as condições ambientais da região com ótimas produtividades em plantios realizados entre novembro e janeiro (OLIBONE et al., 2020).

Estas pesquisas são realizadas na área experimental do Instituto Federal de Educação, Ciência e Tecnologia de Mato Grosso - Campus Sorriso, localizado no município de Sorriso-MT, em parceria com o Programa de Melhoramento do Amendoim (PMA), da Empresa Brasileira de Pesquisa Agropecuária (EMBRAPA), desde a safra 2018/19. Com base nesses estudos de Rizzi et al. (2019) e Rizzi et al. (2020), avaliando características agronômicas das cultivares e linhagens produzidas pelo PMA, os resultados significativos demonstram produtividades superiores à estimativa média nacional da primeira safra $\left(3.679,0 \mathrm{~kg} \mathrm{ha}^{-1}\right)$, segundo CONAB (2021).

Contudo, considerando o regime pluviométrico da região, plantios muito antecipados dificultam a colheita devido ao excesso de umidade, prejudicando na qualidade do produto, tendo em vista que os produtores ainda não possuem estrutura de secagem para amendoim em vagem. Assim, a definição da época ideal de plantio e dos genótipos mais adequados ainda se fazem necessários visando a melhor adequação ao sistema produtivo da região. Dessa forma, a seleção de linhagens de hábitos de crescimento precoces que possibilitam o plantio em segunda safra, após a soja, pode favorecer o crescimento da cultura no estado.

A seleção de linhagens com boa adaptabilidade e estabilidade de produção nas condições ambientais da região é objetivo do Programa de Melhoramento da EMBRAPA e do Instituto Federal de Educação, Ciência e Tecnologia de Mato Grosso - Campus Sorriso, a fim de atender as demandas do mercado interno e expandir as exportações. Essas avaliações exigem muita dedicação, investimento e organização para identificar genitores em potencial e apoiar a tomada de decisões sobre linhagens promissoras para recomendação aos agricultores (RAMALHO et al., 2012). 
Nesse sentido, este trabalho objetivou a avaliação agronômica de genótipos de amendoim no Médio Norte do estado do Mato Grosso.

\section{Material e métodos}

O experimento foi desenvolvido no ano agrícola 2020/21 com semeadura manual no dia 06 de janeiro de 2021, na área experimental do Instituto Federal de Educação, Ciência e Tecnologia de Mato Grosso Campus Sorriso, localizado no município de Sorriso-MT, cujas coordenadas geográficas são $55^{\circ} 48^{\prime} 07^{\prime \prime} \mathrm{W}$ e $12^{\circ} 41^{\prime} 43^{\prime \prime} \mathrm{S}$, com altitude média de $358 \mathrm{~m}$. O solo é classificado como Latossolo Vermelho Amarelo distrófico com textura média. O clima da região é tipo Aw (KÖPPEN; GEIGER, 1928), com temperatura média de $26,2^{\circ} \mathrm{C}$ e pluviosidade média de $1970 \mathrm{~mm}$ anuais os quais são distribuídos nos meses de outubro a abril (SOUZA et al., 2013).

O delineamento experimental foi em blocos casualizados, com quatro repetições. Os tratamentos foram dez genótipos de amendoim rasteiro, duas cultivares: BRS $421 \mathrm{OL}, \mathrm{BRS} 423 \mathrm{OL}$ e oito linhagens: 1253 OL, 2055 OL, 2091 OL, 2101 OL, 2110 OL, 2133 OL, 2136 OL e 2259 OL, desenvolvidas pelo PMA da EMBRAPA. As parcelas foram compostas por duas linhas de três metros de comprimento, espaçamento ente linhas de $0,90 \mathrm{~m}$, intervalo entre parcelas de dois metros e parcela útil 5,4 $\mathrm{m}^{2}$.

Antecedendo a implantação do experimento, foi efetuado o levantamento da fertilidade e granulometria do solo, utilizando-se metodologia proposta por Raij et al. (2001) e Embrapa (1997), na profundidade de 0 a $0,20 \mathrm{~m}$. Os resultados das análises foram: $\mathrm{pH}\left(\mathrm{CaCl}_{2}\right)$ 5,40; P (mehlich) 17,2 $\mathrm{mg} \mathrm{dm}^{-3} ;{\mathrm{K} 70,9 \mathrm{mg} \mathrm{dm}^{-3} \text {; Zn 1,9 mg dm}}^{-3}$; Fe $38 \mathrm{mg}$ $\mathrm{dm}^{-3}$; Mn 4,3 mg dm${ }^{-3}$; Cu 0,6 mg dm${ }^{-3}$; Ca 2,12 $\mathrm{cmolc} \mathrm{dm}^{-3}$; $\mathrm{Mg}$ 0,81 $\mathrm{cmolc}_{\mathrm{c}}$

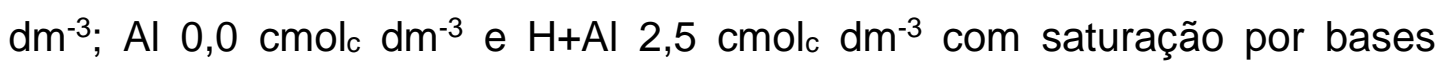
$55,5 \%$ e matéria orgânica 1,94\%. A análise granulométrica de areia, silte e argila apresentou 672,60 e $268 \mathrm{~g} \mathrm{dm}^{-3}$, respectivamente.

O solo foi preparado com a passagem de 2 gradagens pesadas e 1 gradagem com niveladora e em seguida foi realizada a semeadura manual do amendoim, distribuindo-se 18 sementes por metro linear (germinação 
90\%). Em cobertura, aos 35 dias após a emergência (DAE), foi realizada a aplicação de $500 \mathrm{~kg} \mathrm{ha}^{-1}$ de gesso agrícola, aplicado a lanço em área total.

Para o manejo fitossanitário da área experimental foram realizadas seis aplicações, sendo-as nos dias: 05/02/2021 (chlorothalonil + trifloxystrobin + cyproconazole); 21/02/2021 (chlorothalonil + imidacloprid + bifenthrin + indoxacarb + novalurom); 04/03/2021 (chlorothalonil I + trifloxystrobin + cyproconazole + imidacloprid + bifenthrin + indoxacarb + novalurom); 11/03/2021 (chlorothalonil + trifloxystrobin + cyproconazole + indoxacarb + novalurom + chlorfenapyr); 24/03/2021 (chlorothalonil + pyraclostrobin + epoxiconazole); e 03/04/2021 (chlorothalonil + pyraclostrobin + epoxiconazole + chlorfenapyr) utilizando as doses recomendadas pelos fabricantes.

A severidade de mancha preta (Cercosporidium personatum) foi avaliada usando a escala diagramática da incidência com notas de 1 a 9 ao final do ciclo dos genótipos, antes da inversão do experimento (SUBRAHMANYAM et al., 1982). A colheita foi realizada aos 119 dias após o plantio (DAP). Foram avaliados massa de 100 grãos (g) e produtividade de vagens ( $\mathrm{kg} \mathrm{ha}^{-1}$ e sacas alqueire $\left.{ }^{-1}\right)$ mediante a pesagem de vagens e grãosda área total de cada parcela.

A avaliação rendimento (\%) consistiu na retirada de uma amostra aleatória de amendoim em vagens de cada parcela sendo considerado $100 \%$. Após o beneficiamento mecanizado simulando um sistema industrial foi feito a massa de grãos e a casca foi desprezada, com isso calculando a porcentagem de rendimento das parcelas. Os dados obtidos foram submetidos à análise de variância (Teste F) e as médias dos tratamentos foram comparados pelo teste de Scott-Knott a 5\% de probabilidade, por meio do programa computacional SISVAR 5.6 (FERREIRA, 2019).

\section{Resultados e discussão}

Os genótipos apresentaram diferença significativa para severidade de mancha preta (nota), massa de 100 grãos (g), rendimento (\%) e 
produtividade de vagens $\left(\mathrm{kg} \mathrm{ha}^{-1}\right)$ em função de diferentes genótipos de amendoim no Médio Norte do estado de Mato Grosso (Tabela 1).

Na severidade de mancha preta, observa-se as maiores notas nos genótipos 2101 OL (7,8), 2259 OL $(7,7), 2110$ OL $(7,6), 1253$ OL $(7,2)$ e $2055 \mathrm{OL}(7,1)$. Por outro lado, notas intermediárias foram observadas no 2136 OL (6,7), 2133 OL $(6,5)$ e 2091 OL (6,3). Por fim, as menores notas foram obtidas no BRS $423 \mathrm{OL}(5,8)$ e BRS 421 OL $(5,3)$, demonstrando possível tolerância a $C$. personatum em comparação aos demais genótipos (Tabela 1). Corroborando com Rizzi et al. (2020), que obtiveram com o BRS $421 \mathrm{OL}(5,9)$ e BRS $423 \mathrm{OL}(5,7)$ as menores notas, em comparação a outros 6 genótipos, nas condições de Sorriso-MT.

Tabela 1. Severidade de mancha preta (nota), massa de 100 grãos (g), rendimento (\%) e produtividade de vagens $\left(\mathrm{kg} \mathrm{ha}^{-1}\right.$ e sacas alqueire $\left.{ }^{-1}\right)$ em função de diferentes genótipos de amendoim no Médio Norte do estado de Mato Grosso. Sorriso-MT, 2020/21.

\begin{tabular}{|c|c|c|c|c|c|}
\hline \multirow{2}{*}{ Genótipos } & \multirow{2}{*}{$\begin{array}{c}\text { Severidade } \\
\text { (nota) }\end{array}$} & \multirow{2}{*}{$\begin{array}{c}\text { Massa de } \\
100 \text { grãos } \\
\text { (g) }\end{array}$} & \multirow{2}{*}{$\begin{array}{c}\text { Rendimento } \\
(\%)\end{array}$} & \multicolumn{2}{|c|}{ Produtividade de vagens } \\
\hline & & & & $\left(\mathrm{kg} \cdot \mathrm{ha}^{-1}\right)$ & $\left(\right.$ sacas.alqueire $^{-1}$ ) \\
\hline BRS $421 \mathrm{OL}$ & $5,3 a$ & $70,1 \mathrm{a}$ & 79,2 b & $4.744,9 a$ & 459,3 \\
\hline $2101 \mathrm{OL}$ & $7,8 \mathrm{e}$ & $57,1 \mathrm{c}$ & 79,6 b & $4.382,9 \mathrm{a}$ & 424,3 \\
\hline $2259 \mathrm{OL}$ & $7,7 \mathrm{e}$ & $52,0 \mathrm{~d}$ & $79,2 \mathrm{~b}$ & $4.366,2 \mathrm{a}$ & 422,7 \\
\hline $2055 \mathrm{OL}$ & $7,1 d$ & $53,6 \mathrm{c}$ & $82,1 \mathrm{a}$ & $4.309,7 \mathrm{a}$ & 417,2 \\
\hline $1253 \mathrm{OL}$ & $7,2 \mathrm{~d}$ & $61,6 \mathrm{~b}$ & $80,7 \mathrm{a}$ & $4.232,4 \mathrm{a}$ & 409,7 \\
\hline $2136 \mathrm{OL}$ & $6,7 \mathrm{c}$ & $55,9 \mathrm{c}$ & $81,6 \mathrm{a}$ & $4.000,5 b$ & 387,3 \\
\hline BRS $423 \mathrm{OL}$ & $5,8 \mathrm{~b}$ & $57,6 \mathrm{c}$ & $80,7 \mathrm{a}$ & $3.902,8 \mathrm{~b}$ & 377,8 \\
\hline $2133 \mathrm{OL}$ & $6,5 \mathrm{c}$ & $57,3 \mathrm{c}$ & $78,9 \mathrm{~b}$ & $3.765,3 \mathrm{~b}$ & 364,5 \\
\hline $2110 \mathrm{OL}$ & $7,6 \mathrm{e}$ & $48,7 d$ & $80,3 \mathrm{a}$ & $3.747,2$ b & 362,8 \\
\hline 2091 OL & $6,3 c$ & $49,4 d$ & $81,0 \mathrm{a}$ & $3.688,4 b$ & 357,1 \\
\hline Média & 6,8 & 56,3 & 80,3 & $4.114,0$ & 398,3 \\
\hline C.V. (\%) & 4,7 & 4,2 & 1,1 & 10,4 & - \\
\hline $\mathrm{Pr}>\mathrm{Fc}$ & $>0,0001^{*}$ & $>0,0001^{*}$ & $0,0001^{*}$ & $0,0221^{*}$ & - \\
\hline
\end{tabular}

$\mathrm{Na}$ massa de 100 grãos, observa-se que o genótipo BRS 421 OL $(70,1 \mathrm{~g})$ obteve a maior média, resultando na maior granulometria. Pelo contrário, as menores massas foram obtidas no 2110 OL (48,7 g), $2091 \mathrm{OL}$ $(49,4 \mathrm{~g})$ e $2259 \mathrm{OL}(52,0 \mathrm{~g})$, demonstrando massas 31,30 e 26\% inferiores à obtida no BRS 421 OL, respectivamente (Tabela 1). Nesse sentido, 
concorda-se com Rizzi et al. (2019), que observaram massa de 100 grãos correlatas significativamente ao obtido nos genótipos BRS 421 OL e BRS 423 OL, nas condições de Sorriso-MT.

Quanto ao rendimento, os maiores foram observados nos genótipos 2055 OL (82,1\%), 2136 OL (81,6\%), 2091 OL (81,0\%), BRS 423 OL $(80,7 \%)$, 1253 OL $(80,7 \%)$ e 2110 OL (80,3\%), resultando nos genótipos que possuem a espessura da casca mais fina, em comparação aos genótipos 2133 OL (78,9\%), 2259 OL (79,2\%), BRS 421 OL (79,2\%) e 2101 OL $(79,6 \%)$ (Tabela 1). Corroborando com Agulhon et al. (2020), que obtiveram rendimentos semelhantes com o $2091 \mathrm{OL}(78,1 \%)$ e $1253 \mathrm{OL}(78,5)$, nas condições de Santo Antônio do Leste-MT.

As maiores produtividades foram obtidas nos genótipos BRS $421 \mathrm{OL}$ (4.744,9 kg ha-1), $2101 \mathrm{OL}$ (4.382,9 kg ha-1), $2259 \mathrm{OL}$ (4.366,2 kg ha-1), 2055 OL $\left(4.309,7 \mathrm{~kg} \mathrm{ha}^{-1}\right)$ e $1253 \mathrm{OL}\left(4.232,4 \mathrm{~kg} \mathrm{ha}^{-1}\right)$. Em contraparte, os genótipos $2136 \mathrm{OL}\left(4.000,5 \mathrm{~kg} \mathrm{ha}^{-1}\right)$, BRS $423 \mathrm{OL}\left(3.902,8 \mathrm{~kg} \mathrm{ha}^{-1}\right), 2133 \mathrm{OL}$ (3.765,3 $\left.\mathrm{kg} \mathrm{ha}^{-1}\right), 2110 \mathrm{OL}\left(3.747,2 \mathrm{~kg} \mathrm{ha}^{-1}\right)$ e $2091 \mathrm{OL}\left(3.688,4 \mathrm{~kg} \mathrm{ha}^{-1}\right)$ (Tabela 1). A produtividade média do experimento foi de $4.114,0 \mathrm{~kg} \mathrm{ha}^{-1}$, sendo de aproximadamente $12 \%$ superior à estimativa média nacional da CONAB (2021) de amendoim primeira safra $\left(3.679,0 \mathrm{~kg} \mathrm{ha}^{-1}\right)$.

Estudos desenvolvidos com as mesmas configurações de semeadura e testando diferentes genótipos de amendoim produzidos pelo PMA, foram relatados por Xavier et al. (2020), Santin et al. (2020) e Agulhon et al. (2020). Estes resultados reforçam a importância da avaliação, pois ambos autores mencionados obtiveram efeito significativo entre as cultivares e linhagens (genótipos).

\section{Conclusões}

Houve diferença significativa entre os genótipos avaliados para severidade de mancha preta, massa de 100 grãos, rendimento e produtividade. Os menores valores de severidades foram observados nas cultivares BRS $421 \mathrm{OL}$ e BRS $423 \mathrm{OL}$, sendo que as sementes de maior tamanho também foram observadas na BRS 421 OL. As maiores 
produtividades foram obtidas no BRS $421 \mathrm{OL}, 2101 \mathrm{OL}, 2259 \mathrm{OL}, 2055 \mathrm{OL}$ e 1253 OL, com variável tamanho de sementes entre estes genótipos.

\section{Agradecimentos}

Os autores agradecem aos discentes do curso de Engenharia Agronômica do IFMT Campus Sorriso, membros do grupo de pesquisa Sistemas de Produção Agrícola no Cerrado, que contribuíram na condução do experimento. A parceria institucional com o Programa de Melhoramento do Amendoim - Embrapa (vinculado ao projeto SEG 20.18.01.021.00), LC Sementes, Fazenda São José e a MIAC - Indústrias Colombo. 


\section{Referências}

AGULHON, F. S.; HEUERT, J.; MARTINS, K. B. B.; XAVIER, M. F. N.; SUASSUNA, T. M. F. Desempenho agronômico de genótipos de amendoim nas condições de Santo Antônio do Leste-MT. South American Sciences, v. 1, n. 1, p. e2021, 2020.

CONAB. Acompanhamento da Safra Brasileira de Grãos. Safra 2020/21 Nono levantamento, v. 8, n. 9, p. 1-121, 2021. Disponível em: <https://www.conab.gov.br/info-agro/safras>. Acesso em: 27 jun. 2021.

EMBRAPA. Centro Nacional de Pesquisa de Solos (Rio de Janeiro, RJ). Manual de métodos de análise de solo. 2.ed. Rio de Janeiro Embrapa CNPS, 1997.212p

FERREIRA, D. F. SISVAR: um sistema de análise de computador para efeitos fixos projetos de tipo de partida dividida. Revista Brasileira de Biometria, v. 37, n. 4, p. 529-535, 2019.

KÖPPEN, W.; GEIGER, R. Klimate der Erde. Gotha: Verlagcondicionadas. Justus Perthes. 1928.

OLIBONE, D.; WERNER, F. P.; HEUERT, J.; PIVETTA, L. G.; DUARTE, M. M. S.; XAVIER, M. F. N. Desempenho agronômico de cultivares de amendoim em diferentes épocas de semeadura nas condições de SorrisoMT. South American Sciences, v. 1, n. 2, p. e2041, 2020.

RAIJ, B.; ANDRADE, J. C.; CANTARELLA, H.; QUAGGIO, J. A. Análise química para avaliação da fertilidade de solos tropicais. Campinas Instituto Agronômico, 2001. 285p.

RAMALHO, M. A. P.; ABREU, A. F. B.; SANTOS, J. B.; NUNES, J. A. R. Aplicações da genética quantitativa no melhoramento de plantas autógamas. 1. ed. Lavras: UFLA, 2012. 522 p.

RIZZI, T. S.; OLIBONE, D.; HEUERT, J.; XAVIER, M. F. N.; MOURA, G. M.; SUASSUNA, T. M. F. Desempenho agronômico de genótipos de amendoim nas condições de Sorriso-MT. South American Sciences, v. 1, n. 2, p. e2043, 2020.

RIZZI, T. S.; OLIBONE, D.; LODEA, L.; HEUERT, J.; XAVIER, M. F. N.; SUASSUNA, T. M. F. Desempenho de cultivares de amendoim na região médio-norte Mato-grossense. In: Anais do encontro sobre a cultura do amendoim, 16., 2019, Jaboticabal. Anais eletrônicos... Campinas, GALOÁ, 2019.

SANTIN, V.; HEUERT, J.; XAVIER, M. F. N.; RODRIGUES, M. A.; SUASSUNA, T. M. F. Desempenho agronômico de linhagens finais de amendoim nas condições de Campo Verde-MT. South American Sciences, v. 1, n. 1, p. e2011, 2020. 
SOUZA, A. P.; MOTA, L. L.; ZAMADEI, T. MARTIM, C. C.; ALMEIDA, F. T.; PAULINO, J. Classificação climática e balanço hídrico climatológico no estado de Mato Grosso. Nativa, v. 1, n. 1, p. 34-43, 2013.

SUBRAHMANYAM, P.; MCDONALD, D.; GIBBONS, R. W.; NIGAM, S. N.; NEVILL, D. J. Resistance to rust and late leaf spot diseases in some genotypes of Arachis hypogaea. Peanut Science, v. 9, p. 9-14, 1982.

XAVIER, M. F. N.; MEINKE, G. C.; HEUERT, J.; MARTINS, K. B. B.; SUASSUNA, T. M. F. Desempenho agronômico de genótipos de amendoim nas condições de Campo Verde-MT. South American Sciences, v. 1, n. 1, p. e2009, 2020. 